\title{
Estudo Morfométrico do Gênero Panstrongylus Berg, 1879 (Hemiptera, Reduviidae, Triatominae)
}

\author{
Carolina Magalhães dos Santos/ ${ }^{+}$, José Jurberg, Cleber Galvão, Dayse da Silva Rocha, \\ Jaime Ivan Rodriguez Fernandez*
}

\begin{abstract}
Laboratório Nacional e Internacional de Referência em Taxonomia de Triatomíneos, Departamento de Entomologia, Instituto Oswaldo Cruz-Fiocruz, Av. Brasil 4365, 21045-900 Rio de Janeiro, RJ, Brasil * Departamento de Zoologia, Universidade Federal do Paraná, Curitiba, PR, Brasil
\end{abstract}

\begin{abstract}
Morphometric Study of the Genus Panstrongylus Berg, 1879 (Hemiptera, Reduviidae, Triatominae) - The subfamily Triatominae is actually represented by 137 species distributed among 6 tribes and 19 genera. Within this subfamily, the genus Panstrongylus, Berg 1879, is composed by 13 species widespread in sylvatic, peridomestic, and domestic habitats of Neotropical regions. These species are vectors of Chagas disease and consequently are found associated with its main hosts, such as birds and mammals. Interest in species of this genus has been increasing in the last few years. Reports of several authors indicate these Triatominae to invade and colonize houses, increasing their epidemiological significance. Morphometry was used in this study to investigate correlations among possible closely related species. We measured 224 specimens among 13 species through a set of metric variables of the head. The results indicated that the genus Panstrongylus seems to be homogeneous since 10 of the 14 species were shown to be closely related.
\end{abstract}

Key words: Panstrongylus - morphometry - Chagas disease vectors - Triatominae

A subfamília Triatominae está atualmente composta por 137 espécies, onde o gênero Panstrongylus Berg, 1879 é um dos mais numerosos, juntamente com Triatoma Laporte, 1832 e Rhodnius Stal, 1859. Ao todo, são 13 espécies de Panstrongylus: P. chinai (Del Ponte, 1929), $P$. diasi Pinto \& Lent, 1946, P. geniculatus (Latreille, 1811), $P$. guentheri Berg, 1879, P. howardi (Neiva, 1911), P. humeralis (Usinger, 1939), P. lenti Galvão \& Palma, 1968, P. lignarius (Walker, 1873), P. lutzi (Neiva \& Pinto, 1923), P. megistus (Burmeister, 1835), P. rufotuberculatus (Champion, 1899), P. sherlocki Jurberg, Carcavallo \& Lent, 2001 e P. tupynambai Lent, 1942 (Galvão et al. 2003).

Geralmente as espécies que compõem este gênero são encontradas associadas com mamíferos e aves, vivendo em habitats silvestres, domiciliares e peridomiciliares, com hábitos terrestres ou subterrâneos, amplamente distribuídas pela região Neotropical.

Algumas espécies deste gênero merecem uma atenção diferenciada por serem incriminadas como vetores primários, secundários e até mesmo terciários na epidemiologia da doença de Chagas.

Neste contexto citamos P. megistus, por ser a principal espécie vetora em vários estados brasileiros, sendo responsável pela transmissão domiciliar do Trypanosoma cruzi ao homem; P. geniculatus por apresentar uma vasta distribuição geográfica e ser encontrado eventualmente

\footnotetext{
Apoio financeiro: CNPq, convênio 123/97 Funasa/Fiocruz, Fiotec projeto 268, Eclat projeto IC18-CT-960054

${ }^{+}$Autor correspondente. Fax: +55-21-2573.4468. E-mail: carolms@ioc.fiocruz.br

Recebido em 13 de março de 2003

Aceito em 8 de outubro de 2003
}

em domicílios e peridomicílios, é considerada espécie de importância secundária na epidemiologia da doença de Chagas; e P. rufotuberculatus que tem sido encontrado em algumas regiões da Amazônia (Lent \& Wygodzinsky 1979, Schofield 1994, Curto de Casas et al. 1996).

Segundo Lent e Wygodzinsky (1979), existe uma gama de hipóteses a serem analisadas neste gênero, como a variação populacional de $P$. rufotuberculatus, ressaltando padrões de coloração e caracteres morfológicos.

A variação populacional existente em $P$. geniculatus também é outro ponto a ser estudado pois a maioria dos espécimes desta espécie apresenta olhos grandes, com exceção de uma população proveniente do Valle de la Convención (Peru), onde os olhos são notadamente menores. Esta variabilidade pode estar relacionada com a distribuição geográfica desta espécie.

A estreita relação entre $P$. humeralis e $P$. lignarius é outra questão interessante, uma vez que Carcavallo et al. (2000) citam estas duas espécies como formadoras do "complexo lignarius".

P. tupynambai, em sua descrição original foi comparado a $P$. guentheri mas, atualmente, do ponto de vista dos estudos sobre estruturas fálicas, está considerado mais próximo de $P$. lutzi devido à presença de dentículos no processo lateral do endosoma que, por sua vez, estão ausentes em P. guentheri (Lent \& Jurberg 1975).

Em relação a $P$. sherlocki, a mais recente espécie descrita, Jurberg et al. (2001) descrevem-na como espécie afim de P. lutzi, devido a suas características morfológicas externas e fálicas, e sugerem a futura composição de um complexo específico formado por P. sherlocki, P. lutzi e P. diasi.

Visto que as espécies de maior importância epidemiológica são justamente aquelas que colonizam os domicílios, trabalhos recentes ressaltam e enfocam o aumento da freqüência com que algumas espécies do gênero têm invadido domicílios, colonizando-os, como $P$. 
rufotuberculatus (Noireau et al. 1994, Dujardin et al. 1998, Salomón et al. 1999), P. geniculatus (Torres et al. 1997, Cabello \& Galíndez 1998, Valente 1999), P. lutzi (Garcia \& Diotaiuti 2001) e P. chinai (Pollack et al. 2001)

O gênero Panstrongylus tem sido estudado por diversos autores sob os mais variados enfoques, valendo ressaltar a genitália do macho (Lent \& Jurberg 1968, 1975), o ciclo evolutivo (Lent \& Jurberg 1969), estudos populacionais a partir de eletroforese de enzimas (Doréa et al. 1982), microscopia eletrônica de varredura da região óculo-ocelar (Carcavallo et al. 1994) e filogenia molecular (Hypsa et al. 2002, Marcilla et al. 2002).

No entanto, poucos trabalhos referentes à morfometria deste gênero têm sido desenvolvidos, cabendo destacar estudos de folículos testiculares de algumas espécies de triatomíneos, dentre elas P. megistus (Gonçalves et al. 1987), estudos com populações de $P$. geniculatus (Torres et al. 1997), estudos populacionais envolvendo P. rufotuberculatus domésticos originários da Bolívia (Dujardin et al. 1998), investigação das espécies que compõem o complexo lignarius (Santos et al. 2001), estudos com populações de P. lutzi do Ceará (Sousa et al. 2001) e populações de P. chinai provenientes do Peru (Pollack et al. 2001),

Tendo em vista a grande diversidade de pontos a serem investigados dentro deste gênero, recorremos à morfometria de parâmetros cefálicos com a finalidade de caracterizar espécies e correlacioná-las com dados já conhecidos sobre similaridades e desigualdades de parâmetros fálicos, isoenzimáticos e ecológicos e assim contribuir tanto para a análise sistemática como para a análise filogenética.

\section{MATERIAIS E MÉTODOS}

Foram utilizados espécimes adultos depositados nas coleções "Herman Lent" e "Rodolfo U. Carcavallo" do Laboratório Nacional e Internacional de Referência em Taxonomia de Triatomíneos (LNIRTT) do Instituto Oswaldo Cruz-Fiocruz, Rio de Janeiro, Brasil.

Morfometria - Foram medidos 224 espécimes de diferentes localidades distribuídos entre as 13 espécies exis- tentes. Foi incluída ainda uma população até pouco tempo descrita como P. herreri Wygodzinsky, 1948 e recentemente sinonimizada como $P$. lignarius por Marcilla et al. (2002) (Tabela I).

O método utilizado foi a morfometria tradicional que se baseia na distância entre pontos de referência (variáveis) que melhor definem a "silhueta" da cabeça (Dujardin 1999).

Primeiramente todas as distâncias foram tomadas na objetiva de $25 \mathrm{X}$ e ocular de $10 \mathrm{X}$, em um microscópio estereoscópico M5-Wild, com auxílio de uma lente ocular graduada (Zeiss). Posteriormente, as distâncias em unidades de graduação foram convertidas em milímetros e logarítmos naturais, de acordo com a exigência do teste estatístico empregado.

As variáveis determinadas e utilizadas são consideradas por Lent e Wygodzinsky (1979) como importantes na taxonomia dos Triatominae: CTC - comprimento total da cabeça; RO - largura da cabeça ao nível da região ocular; RAO - comprimento da região ante-ocular; RPO - comprimento da região pós-ocular; RIA - comprimento da região inter-ocular; RIE - comprimento da região inter-ocelar; COL - comprimento do olho em vista lateral; COD - comprimento do olho em vista dorsal; TA - tubérculo antenífero; AC - largura do anteclípeo; R1 - comprimento do $1^{\circ}$ segmento do rostro; R2 - comprimento do $2^{\circ}$ segmento do rostro; R3 - comprimento do $3^{\circ}$ segmento do rostro (Fig. 1).

Análise e estatística - Primeiramente foi realizada a análise quantitativa dos dados morfométricos (Tabela II). Posteriormente estes dados foram estudados com o emprego da análise de componentes principais (ACP) onde montamos um gráfico bidimensional a partir dos dois primeiros componentes principais para ilustrar nossos resultados (Dujardin 1999).

Para verificar as diferenças significativas entre os grupos, utilizamos os resultados provenientes da ACP, especificamente os valores de CP1 e CP2, para avaliar estas diferenças usando o software JMP.

A análise de cluster que gerou o dendrograma foi baseada nos valores médios padronizados obtidos pela ACP de cada espécie; com este resultado calculamos as dis-

TABELA I

Distribuição geográfica dos espécimes de Panstrongylus analisados

\begin{tabular}{|c|c|c|c|c|c|c|c|c|c|c|c|}
\hline Espécies/Procedência & $\mathrm{Lab}$ & $\mathrm{Pe}$ & Ve & $\mathrm{Br}$ & $\mathrm{Eq}$ & Ar & Pg & $\mathrm{Gu}$ & Pn & Bo & Total \\
\hline P. chinai & 1 & 6 & 1 & & 6 & & & & & & 14 \\
\hline P. diasi & & & & 2 & & & & & & & 2 \\
\hline P. geniculatus & & & & 30 & & & & & & & 30 \\
\hline P. guentheri & & & & & & 6 & 3 & & & & 9 \\
\hline P. herreri ${ }^{a}$ & 30 & & & & & & & & & & 30 \\
\hline P. howardi & & & & & 1 & & & & & & 1 \\
\hline P. humeralis & 19 & & & & & & & 3 & 2 & & 24 \\
\hline P. lenti & & & & 2 & & & & & & & 2 \\
\hline P. lignarius $^{a}$ & 30 & & & & & & & & & & 30 \\
\hline P. lutzi & & & & 22 & & & & & & & 22 \\
\hline P. megistus & 30 & & & & & & & & & & 30 \\
\hline P. rufotuberculatus & & & 3 & & & & & & 1 & 22 & 26 \\
\hline P. sherlocki & & & & 1 & & & & & & & 1 \\
\hline P. tupynambai & & & & 3 & & & & & & & 3 \\
\hline
\end{tabular}

Lab: criação de laboratório; Pe: Peru; Ve: Venezuela; Br: Brasil; Eq: Equador; Ar: Argentina; Pg: Paraguai; Gu: Guiana; Pn: Panamá; Bo: Bolívia; $a$ : P. herreri tornou-se sinonímia de P. lignarius por Marcilla et. al (2002). 

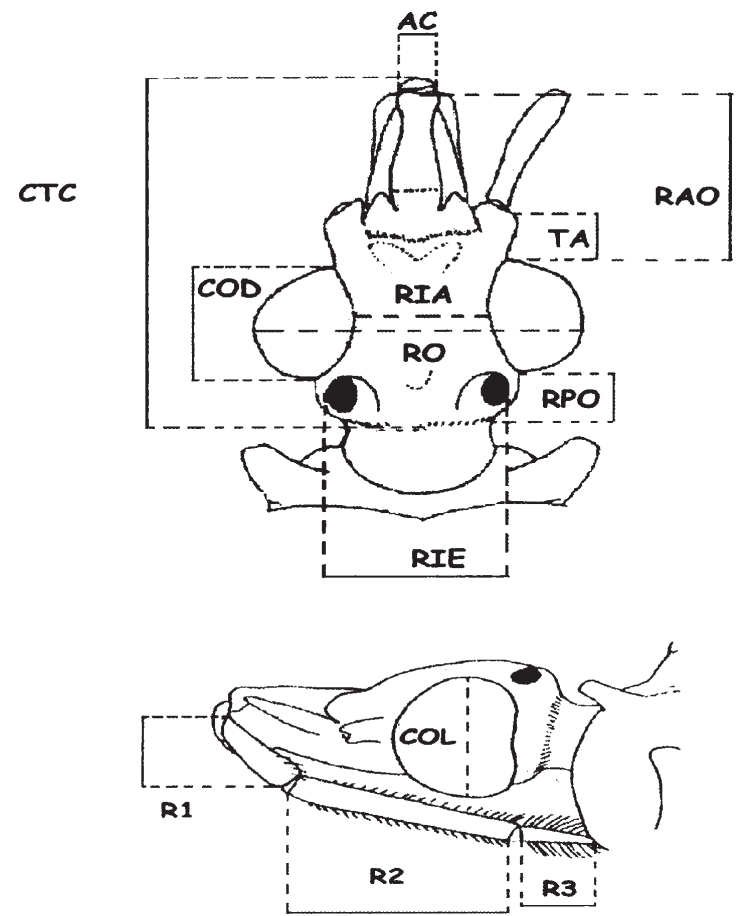

Fig. 1: vista dorsal e lateral, respectivamente, da cabeça de um Panstrongylus sp. com indicação dos 13 parâmetros utilizados na morfometria. CTC: comprimento total da cabeça; RO: largura da cabeça ao nível da região ocular; RAO: região ante-ocular; RIA: região inter-ocular; RIE: região inter-ocelar; RPO: região pós-ocular; TA: tubérculo antenífero; AC: anteclípeo; COD: comprimento do olho em vista dorsal; COL: comprimento do olho em vista lateral; R1: primeiro segmento do rostro; R2: segundo segmento do rostro; R3: terceiro segmento do rostro (Figura adaptada de Dujardin 1999).

tâncias taxonômicas médias entre as espécies do gênero. Todas as análises foram feitas com o software JMP (SAS Institute 1995).

\section{RESULTADOS}

Através da estatística descritiva das 13 variáveis métricas em cada espécie ressaltamos que os valores referentes ao desvio-padrão das variáveis em $P$. howardi e $P$. sherlocki são nulos, pois as respectivas espécies contaram com apenas um espécime representativo. De acordo com os resultados obtidos observamos que $P$. lenti é menor que as demais espécies, pois apresenta valores relativamente menores em praticamente todas as variáveis medidas na cabeça. No entanto $P$. megistus, $P$. rufotuberculatus e $P$. sherlocki possuem valores mais elevados, sendo consideradas espécies com dimensões de cabeça maiores (Tabela II).

Com a finalidade de ajustar o tamanho da amostra ao número de variáveis utilizadas e pelo fato das demais variáveis esboçarem de forma mais abrangente o perfil dos insetos, algumas foram eliminadas: RPO, RIA, RIE, AC, R2, COL e COD (Pimentel 1992). Assim, as análises seguintes foram realizadas com base nas seis variáveis restantes: CTC, RO, RAO, TA, R1 e R3.

Os resultados da ACP para comparar as espécies nos mostraram que os dois primeiros componentes principais $(\mathrm{CP} 1=52,9 \%$ e $\mathrm{CP} 2=22,1 \%)$ juntos correspondem a $75 \%$

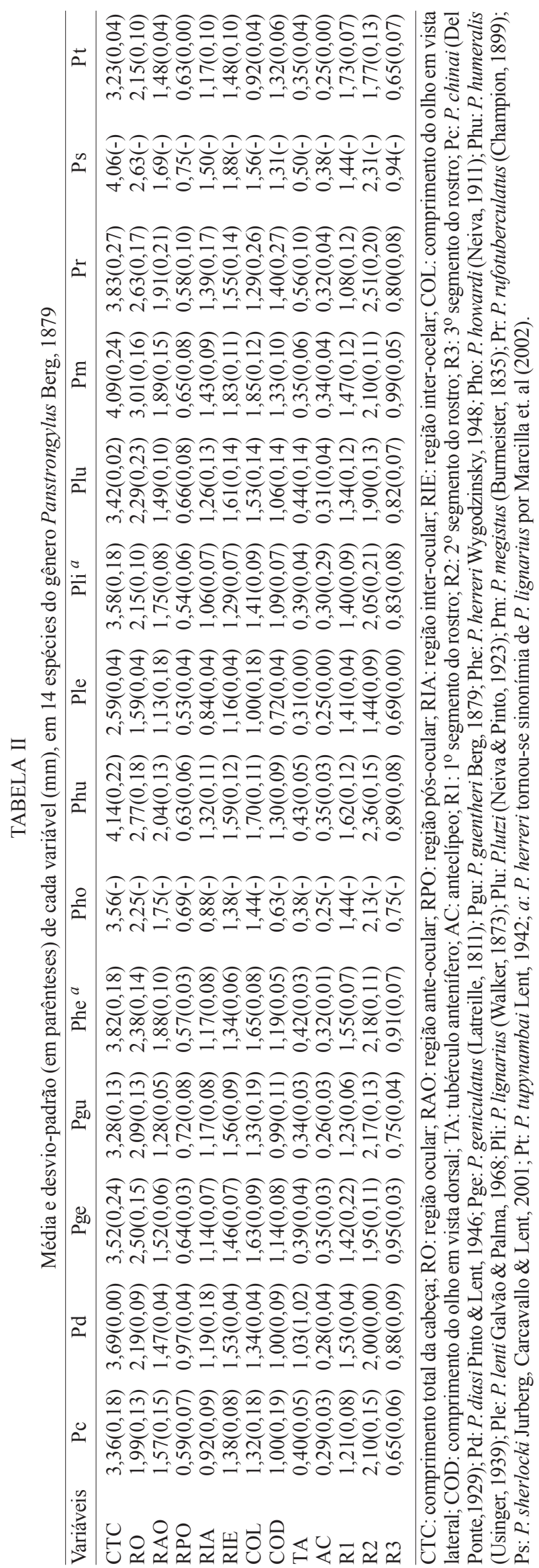


da variabilidade total; a partir destes dados foi montado um mapa bidimensional (CP1 X CP2), onde podemos observar nitidamente a separação de $P$. diasi, $P$. lenti, $P$. rufotuberculatus e $P$. tupynambai das demais espécies que, por sua vez, não apresentam uma boa diferenciação (Fig. 2).

Nas Tabelas III (valores de CP1) e IV (valores de CP2) os pares de espécies significativamente diferentes apresentam valores positivos.

Em relação ao resultado demonstrado através do dendrograma (Fig. 3), fica evidente que as amostras de $P$. herreri e $P$. lignarius são bastante similares, uma vez que ocupam o mesmo cluster e, ainda, apresentam as menores distâncias dentro de todo o grupo. O mesmo ocorre com P. lutzi e P. sherlocki, P. lenti e P. tupynambai, porém com distâncias respectivamente maiores. $P$. rufotuberculatus e $P$. diasi demonstraram serem as espécies mais distantes dentro do gênero.

\section{DISCUSSÃO}

A variabilidade morfométrica exibida na análise de componentes principais indica que o gênero Panstrongylus é bastante homogêneo, com algumas ressalvas como $P$. diasi, $P$. lenti, $P$. rufotuberculatus e $P$. tupynambai. Embora a variabilidade fenotípica seja influenciada por diversos fatores como clima, procedência geográfica,

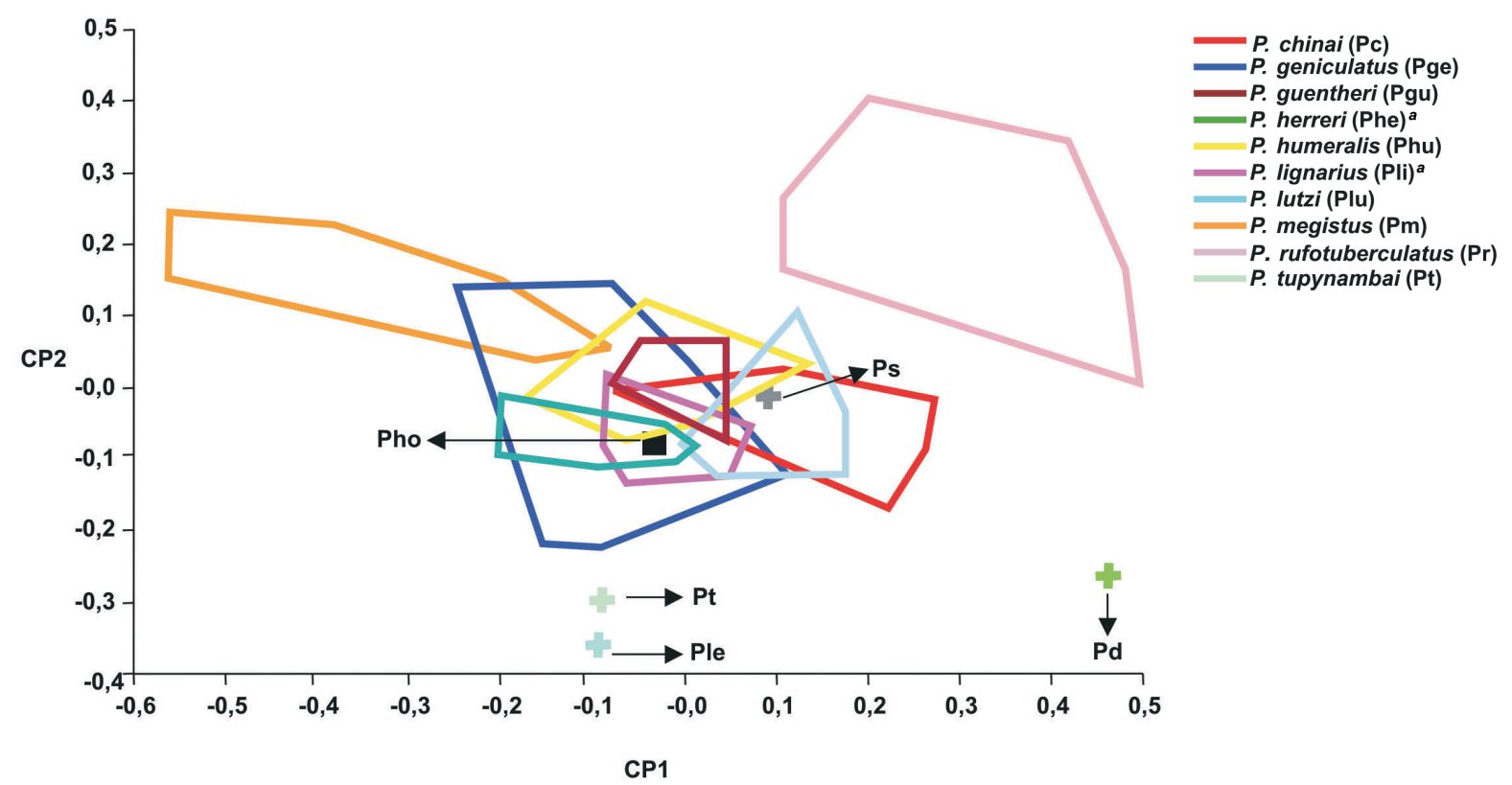

Fig. 2: mapa fatorial (CP1 X CP2) entre as espécies de Panstrongylus. CP1: primeiro componente principal (52,9\%); CP2: segundo componente principal $(22,1 \%)$ demonstrando $75 \%$ da variação total. Pd: P. diasi; Pho: P. howardi; Ple: P. lenti; Ps: P. sherlocki; Pt: P. tupynambai; a: P. herreri tornou-se sinonímia de P. lignarius por Marcilla et. al (2002).

TABELA III

Comparação aos pares com as espécies do gênero Panstrongylus Berg, 1879, com os valores do primeiro componente principal

\begin{tabular}{|c|c|c|c|c|c|c|c|c|c|c|c|c|c|c|}
\hline & $\mathrm{Pd}$ & $\operatorname{Pr}$ & $\mathrm{Pc}$ & Ps & Plu & Phu & $\mathrm{Pli}^{a}$ & Pho & Ple & Pgu & $\mathrm{Phe}^{a}$ & $\mathrm{Pt}$ & Pge & $\mathrm{Pm}$ \\
\hline \multicolumn{15}{|l|}{$\mathrm{Pd}$} \\
\hline $\operatorname{Pr}$ & $-0,184$ & & & & & & & & & & & & & \\
\hline Pc & 0,003 & $-0,015$ & & & & & & & & & & & & \\
\hline Ps & $-0,201$ & $-0,304$ & $-0,202$ & & & & & & & & & & & \\
\hline Plu & 0,039 & 0,020 & $-0,491$ & $-0,504$ & & & & & & & & & & \\
\hline Phu & 0,161 & 0,142 & $-0,167$ & $-0,382$ & $-0,080$ & & & & & & & & & \\
\hline $\mathrm{Pli}^{a}$ & 0,165 & 0,147 & $-0,045$ & $-0,378$ & $-0,076$ & $-0,198$ & & & & & & & & \\
\hline Pho & $-0,050$ & $-0,153$ & $-0,040$ & $-0,549$ & $-0,375$ & $-0,497$ & $-0,502$ & & & & & & & \\
\hline Ple & 0,072 & $-0,005$ & $-0,192$ & $-0,445$ & $-0,228$ & $-0,350$ & $-0,354$ & $-0,596$ & & & & & & \\
\hline Pgu & 0,182 & 0,156 & $-0,031$ & $-0,358$ & $-0,066$ & $-0,188$ & $-0,193$ & $-0,510$ & $-0,385$ & & & & & \\
\hline $\mathrm{Phe}^{a}$ & 0,200 & 0,182 & $-0,005$ & $-0,343$ & $-0,041$ & $-0,163$ & $-0,167$ & $-0,494$ & $-0,367$ & $-0,209$ & & & & \\
\hline $\mathrm{Pt}$ & 0,146 & 0,084 & $-0,103$ & $-0,379$ & $-0,139$ & $-0,261$ & $-0,265$ & $-0,531$ & $-0,422$ & $-0,032$ & $-0,300$ & & & \\
\hline Pge & 0,229 & 0,213 & 0,256 & $-0,314$ & $-0,010$ & $-0,132$ & $-0,136$ & $-0,466$ & $-0,338$ & $-0,179$ & $-0,171$ & $-0,310$ & & \\
\hline $\mathrm{Pm}$ & 0,443 & 0,424 & 0,237 & $-0,100$ & 0,202 & 0,080 & 0,076 & $-0,251$ & $-0,125$ & 0,034 & 0,041 & $-0,096$ & 0,017 & \\
\hline
\end{tabular}

Os valores positivos mostram pares de espécies significativamente diferentes. Pc: P. chinai (Del Ponte,1929); Pd: P. diasi Pinto \& Lent, 1946; Pge: P. geniculatus (Latreille, 1811); Pgu: P. guentheri Berg, 1879; Phe: P. herreri Wygodzinsky, 1948; Pho: P. howardi (Neiva, 1911); Phu: P. humeralis (Usinger, 1939); Ple: P. lenti Galvão \& Palma, 1968; Pli: P. lignarius (Walker, 1873); Plu: P. lutzi (Neiva \& Pinto, 1923); Pm: P. megistus (Burmeister, 1835); Pr: P. rufotuberculatus (Champion, 1899); Ps: P. sherlocki Jurberg, Carcavallo \& Lent, 2001; Pt: P. tupynambai Lent, 1942; a: P. herreri tornou-se sinonímia de P. lignarius por Marcilla et. al (2002). 
TABELA IV

Comparação aos pares com as espécies do gênero Panstrongylus Berg, 1879, com os valores do segundo componente principal

\begin{tabular}{|c|c|c|c|c|c|c|c|c|c|c|c|c|c|c|}
\hline & $\mathrm{Pd}$ & $\mathrm{Pr}$ & $\mathrm{Pc}$ & Ps & Plu & Phu & $\mathrm{Pli}^{a}$ & Pho & Ple & Pgu & $\mathrm{Phe}^{a}$ & $\mathrm{Pt}$ & Pge & $\mathrm{Pm}$ \\
\hline \multicolumn{15}{|l|}{ Ple } \\
\hline $\mathrm{Pt}$ & $-0,153$ & & & & & & & & & & & & & \\
\hline $\mathrm{Pd}$ & $-0,124$ & $-0,173$ & & & & & & & & & & & & \\
\hline $\mathrm{Pli}^{a}$ & 0,112 & 0,070 & $-0,009$ & & & & & & & & & & & \\
\hline $\mathrm{Pgu}$ & 0,110 & 0,068 & $-0,011$ & $-0,105$ & & & & & & & & & & \\
\hline Pho & 0,006 & $-0,048$ & $-0,115$ & $-0,248$ & $-0,254$ & & & & & & & & & \\
\hline Phe $^{a}$ & 0,119 & 0,078 & $-0,002$ & $-0,093$ & $-0,103$ & $-0,254$ & & & & & & & & \\
\hline Plu & 0,126 & 0,085 & 0,005 & $-0,086$ & $-0,096$ & $-0,247$ & $-0,093$ & & & & & & & \\
\hline $\mathrm{Pc}$ & 0,148 & 0,107 & 0,028 & $-0,063$ & $-0,074$ & $-0,225$ & $-0,071$ & $-0,078$ & & & & & & \\
\hline Ps & 0,056 & 0,003 & $-0,065$ & $-0,198$ & $-0,204$ & $-0,296$ & $-0,205$ & $-0,212$ & $-0,099$ & & & & & \\
\hline Pge & 0,191 & 0,149 & 0,070 & $-0,020$ & $-0,031$ & $-0,183$ & $-0,028$ & $-0,035$ & $-0,057$ & $-0,233$ & & & & \\
\hline Phu & 0,195 & 0,154 & 0,075 & $-0,016$ & $-0,027$ & $-0,178$ & $-0,024$ & $-0,031$ & $-0,053$ & $-0,228$ & $-0,092$ & & & \\
\hline $\mathrm{Pm}$ & 0,291 & 0,249 & 0,170 & 0,079 & 0,069 & $-0,083$ & 0,072 & 0,065 & 0,043 & $-0,133$ & 0,003 & $-0,005$ & & \\
\hline $\mathrm{Pr}$ & 0,417 & 0,376 & 0,296 & 0,206 & 0,195 & 0,044 & 0,198 & 0,191 & 0,169 & $-0,006$ & 0,130 & 0,122 & 0,027 & \\
\hline
\end{tabular}

Os valores positivos mostram pares de espécies significativamente diferentes. Pc: P. chinai (Del Ponte,1929); Pd: $P$. diasi Pinto \& Lent, 1946; Pge: P. geniculatus (Latreille, 1811); Pgu: P. guentheri Berg, 1879; Phe: P. herreri Wygodzinsky, 1948; Pho: P. howardi (Neiva, 1911); Phu: P. humeralis (Usinger, 1939); Ple: P. lenti Galvão \& Palma, 1968; Pli: P. lignarius (Walker, 1873); Plu: P. lutzi (Neiva \& Pinto, 1923); Pm: P. megistus (Burmeister, 1835); Pr: P. rufotuberculatus (Champion, 1899); Ps: P. sherlocki Jurberg, Carcavallo \& Lent, 2001; Pt: P. tupynambai Lent, 1942; a: P. herreri tornou-se sinonímia de P. lignarius por Marcilla et. al (2002).

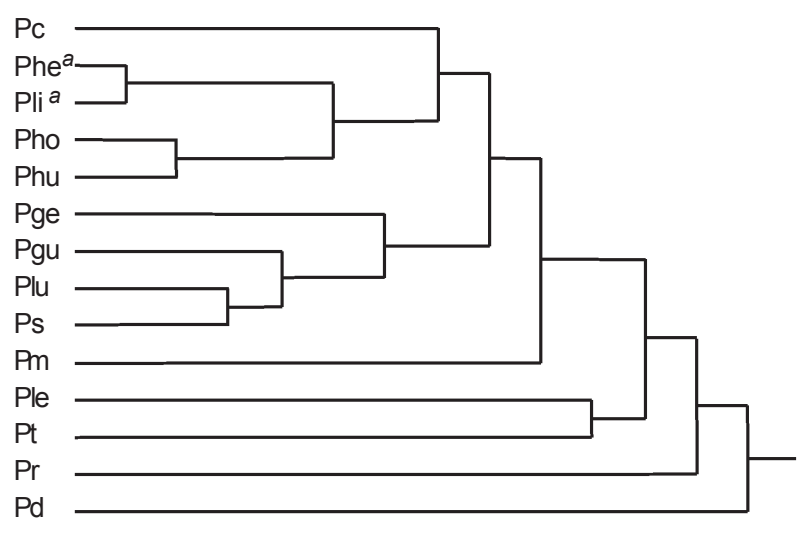

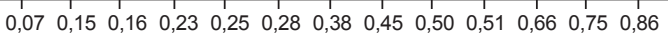

Fig. 3: distâncias taxonômicas médias entre as espécies de Panstrongylus. Valores correspondentes às distâncias taxonômicas médias entre as espécies de Panstrongylus Berg, 1879. Pc: P. chinai (Del Ponte, 1929); Pd: P. diasi Pinto \& Lent, 1946; Pge: P. geniculatus (Latreille, 1811); Pgu: P. guentheri Berg, 1879; Phe: P. herreri Wygodzinsky, 1948; Pho: P. howardi (Neiva, 1911); Phu: P. humeralis (Usinger, 1939); Ple: P. lenti Galváo \& Palma, 1968; Pli: P. lignarius (Walker, 1873); Plu: P. lutzi (Neiva \& Pinto, 1923); Pm: P. megistus (Burmeister, 1835); Pr: P. rufotuberculatus (Champion, 1899); Ps: P. sherlocki Jurberg, Galvão \& Lent, 2001; Pt: P. tupynambai Lent, 1942; a: P. herreri tornou-se sinonímia de P. lignarius por Marcilla et. al (2002).

manutenção em condições de laboratório e oferta de alimento, os resultados refletiram a posição taxonômica já estabelecida por outros autores (Lent \& Jurberg 1975, Lent \& Wygodzinsky 1979, Carcavallo et al. 1994, 2000, Marcilla et al. 2002, Galvão et al. 2003).

Lent e Jurberg (1975), Lent e Wygodzinsky (1979) e Carcavallo et al. (1994), ao estudarem todas as espécies do gênero usando diferentes parâmetros, chegaram à mesma conclusão quanto à relação de $P$. herreri, $P$. humeralis e $P$. lignarius, como espécies que mostram uma estreita relação de parentesco.

Porém, Carcavallo et al. (2000) discutem a possibilidade de $P$. lignarius e $P$. herreri estarem mais relacionadas entre si do que em relação a $P$. humeralis, constituindo assim o "complexo lignarius". No entanto, Marcilla et al. (2002) ressaltam que $P$. lignarius e $P$. herreri são espécies idênticas de acordo com resultados obtidos pela análise de DNA ribossomal (rDNA), sugerindo tratar-se de apenas uma espécie onde $P$. herreri seria sinonímia de $P$. lignarius. Galvão et al. (2003) aceitaram a sinonímia proposta ao publicarem uma lista de espécies válidas da subfamília Triatominae, já incluindo $P$. herreri como sinonímia de $P$. lignarius.

De acordo com a morfometria da região cefálica, a amostra de $P$. herreri se apresenta mais relacionada com $P$. lignarius e distante de $P$. humeralis, corroborando com a sinonímia proposta por Marcilla et al. (2002).

Outro ponto a ser discutido é a posição de $P$. rufotuberculatus, que em diversos trabalhos (Lent \& Jurberg 1975, Lent \& Wygodzinsky 1979, Carcavallo et al. 1994, Marcilla et al. 2002) encontra-se em destaque, separado das demais espécies, o que foi comprovado em nosso trabalho. Esta diferenciação já tinha sido observada anteriormente quando foi criado o gênero Mestor (Kirkhaldy, 1904) para designar P. rufotuberculatus, considerado um gênero à parte, merecendo inclusive uma revisão de Lent e Pífano (1940), fato que precisa ser esclarecido por estudos bioquímicos.

Recentemente, Hypsa et al. (2002) ao estudarem o DNA mitocondrial de quatro espécies chamaram a atenção para a dificuldade da formação de um clado monofilético entre as espécies do gênero Panstrongylus. Posteriormente, Marcilla et al. (2002) ao analisarem cinco espécies utilizando ITS-2 demonstraram o polifiletismo do gênero. No entanto, ambos os autores expõem a necessidade de um estudo mais profundo, utilizando um maior número de 
espécies do gênero a fim de corroborar os resultados obtidos.

A morfometria é uma técnica extremamente sensível e por esta razão é necessário ter muita cautela com o tamanho das amostras utilizadas, uma vez que estas podem gerar erros de leitura. No entanto, apesar de neste estudo termos utilizado algumas espécies com tamanhos amostrais não muito adequados optamos por inseri-las justamente para termos uma visão total e não parcial do gênero. Por esta razão, é prematuro afirmar o isolamento de $P$. diasi, $P$. lenti e P. tupynambai dentro do gênero pois é possível que o tamanho da amostra tenha influenciado no resultado final. Sendo assim, é mais prudente fazer novas análises, quando houver material disponível, com uma amostra mais adequada com a finalidade de obtermos um resultado mais consistente.

\section{AGRADECIMENTOS}

Ao Dr. Jean Pierre Dujardin pelas sugestões, disponibilidade e esclarecimentos imprescindíveis; ao Dr. Rodolfo U Carcavallo pela leitura crítica deste manuscrito.

\section{REFERÊNCIAS}

Cabello DR, Galíndez Girón I 1998. Vital statistics of Panstrongylus geniculatus (Latreille, 1811) (Hemiptera: Reduviidae) under experimental conditions. Mem Inst Oswaldo Cruz 93: 257-262.

Carcavallo RU, Galíndez-Gíron I, Jurberg J, Martínez A 1994. La región óculo-ocelar en género Panstrongylus Berg, 1879 (Hemip-tera, Reduviidae, Triatominae). Entomol Vect 1: 113120.

Carcavallo RU, Jurberg J, Lent H, Noireau F, Galvão C 2000. Phylogeny of the Triatominae (Hemiptera:Reduviidae) proposals for taxonomic arrengements. Entomol Vect 7 (Suppl.1): 1-99.

Curto de Casas SI, Carcavallo RU, Galíndez-Girón I, Jurberg J, Mena-Segura CA 1996. Geographical distribution and altilatitudinal dispersion of species of Panstrongylus (Hemiptera, Reduviidae, Triatominae, Triatomini). Entomol Vect 3:43-58.

Dórea RCC, Póvoa MM, Miles MA, Souza AAA, Barata JM 1982. Eletroforese de enzimas para estudos de triatomíneos com referência especial a subpopulações de Panstrongylus megistus. Rev Brasil Biol 42: 521-526.

Dujardin JP 1999. Introducción a la Morfometria con Énfasis en Triatominae y Phlebotominae. http://eclat.fcien.edu.uy/

Dujardin JP, Forgues G, Torrez M, Martinez E, Cordobá C, Gianella A 1998. Morphometrics of domestic Panstrongylus rufotuberculatus in Bolivia. Ann Med Parasitol 92: 219-228.

Galvão C, Carcavallo RU, Rocha DS, Jurberg J 2003. A checklist of the current valid species of the subfamily Triatominae Jeannel, 1919 (Hemiptera, Reduviidae) and their geographical distribution, with nomenclatural and taxonomic notes. Zootaxa 202: 1-36.

Garcia MHM, Diotaiuti L 2001. Ocorrência do Panstrongylus lutzi no estado do Ceará. Rev Soc Bras Med Trop 34 (Supl. I): 137-138.

Gonçalves TCM, Lent H, Almeida J R 1987. Estudo anatômico e morfométrico dos folículos testiculares de algumas espécies de Triatominae (Hemiptera: Reduviidae). Mem Inst Oswaldo Cruz 82: 543-550.

Hypsa V, Tietz DF, Zrzavý J, Rego ROM, Galvão C, Jurberg J 2002. Phylogeny and biogeography of Triatominae (Hemiptera: Reduviidae): molecular evidence of a New World origin of the Asiatic clade. Mol Phylogenet Evol 23: 447-457.
Jurberg J, Carcavallo RU, Lent H 2001. Panstrongylus sherlocki sp.n. do estado da Bahia, Brasil (Hemiptera, Reduviidae, Triatominae). Entomol Vect 8: 261-274.

Lent H, Jurberg J 1968. Estudo morfológico comparativo de "Panstrongylus geniculatus" (Latreille, 1811) e "Panstrongylus megistus" (Burmeister, 1835) e suas genitálias externas (Hemiptera, Reduviidae, Triatominae). Rev Brasil Biol 28: 499-520.

Lent H, Jurberg J 1969. Observações sobre o ciclo evolutivo, em laboratório, do "Panstrongylus geniculatus" (Latreille, 1811) (Hemiptera, Reduviidae, Triatominae). An Acad Brasil Ciênc 41: 125-131.

Lent H, Jurberg J 1975. O gênero Panstrongylus Berg, 1879, com um estudo sobre a genitália externa das espécies (Hemiptera, Reduviidae, Triatominae). Rev Brasil Biol 35: 379-438.

Lent H, Pifano CF 1940. Sobre a identidade dos gêneros Panstrongylus Berg, 1879 e Mestor Kirkaldy, 1904. Redescrição de Panstrongylus rufotuberculatus encontrado na Venezuela naturalmente infestado pelo Schizotrypanum cruzi. Rev Entomol 11: 629-639.

Lent H, Wygodzinsky P 1979. Revision of the Triatominae (Hemiptera, Reduviidae), and their significance as vectors of Chagas' disease. Bull Am Mus Nat \& History 163: Article 3.

Marcilla A, Barguez MD, Abad-Franch F, Panzera F, Carcavallo RU, Noireau F, Galvão C, Jurberg J, Miles MA, Dujardin JP, Mas-Coma S 2002. Nuclear rDNA ITS-2 reveal polyphyly of Panstrongylus species (Hemiptera: Reduviidae: Triatominae), vectors of Trypanosoma cruzi. Infection, Genet Evol 1: 225-235.

Noireau F, Bosseno MF, Vargas F, Breniére SF 1994. Apparent trend to domesticity observed in Panstrongylus rufotuberculatus Champion, 1899 (Hemiptera:Reduviidae) in Bolivia. Res and Rev in Parasitol 54: 263-264.

Pimentel RA 1992. An introduction to ordination, principal components analysis and discriminant analysis. In RG Foottit, JT Sorensen (eds), Ordination Quantitative Genetic Rationales, Elsevier, New York, p. 11-28.

Pollack V, Ampuero RC, Roldán RJT, Vargas VF, Cuba Cuba CA 2001. Morphometrics and artificial ecotopes of Panstrongylus chinai (Hemiptera, Reduviidae) in Northern Peru. J Brasil Patol 37: 188.

Salomón OD, Ripoll CM, Rivetti E, Carcavallo RU 1999. Presence of Panstrongylus rufotuberculatus (Champion, 1899) (Hemiptera:Reduviidae: Triatominae) in Argentina. Mem Inst Oswaldo Cruz 94: 285-288.

Santos CM, Jurberg J, Galvão C, Galíndez Girón I, Carcavallo RU 2001. Análise morfométrica do "complexo lignarius" (Hemiptera, Reduviidae). J Brasil Patol 37: 170.

SAS Institute 1995. JMP, Statistics and Graphics Guide, Version 3.1, SAS Inc., Cary, NC, 593 pp.

Schofield C 1994. Triatominae Biologia \& Control, Eurocomunica Publications, UK, 77 pp.

Sousa LC, Borges EC, Souza RCM, Abrão DO, Garcia MHM, Diotaiuti L 2001. Morfometria do Panstrongylus lutzi Neiva, Pinto, 1923 (Hemiptera: Reduviidae:Triatominae) no estado do Ceará. Rev Soc Bras Med Trop 34 (Supl. I): 135-136.

Torres E, Galíndez-Girón I, Matos E, Araújo V, Torres A, Barazarte R, Marquez J 1997. Multivariate analysis applied to some populations of Panstrongylus geniculatus (Latreille, 1811) (Hemiptera, Reduviidae, Triatominae). Entomol Vect 4: 191-202.

Valente VC 1999. Potential for domestication of Panstrongylus geniculatus (Latreille, 1811) (Hemiptera:Reduviidae: Triatominae) in the municipality of Muaná, Marajó island, state of Pará, Brazil. Mem Inst Oswaldo Cruz 94 (Suppl. I): 390-400. 